Жантимиров Павел Амамбаевич

студент Департамента общественных финансов Финансового университета

при Правительстве Российской Федерации

\section{УПРАВЛЕНИЕ ОСТАТКАМИ СРЕДСТВ НА ЕДИНОМ СЧЕТЕ ФЕДЕРАЛЬНОГО БЮДЖЕТА В УСЛОВИЯХ ПРОФИЦИТА ЛИКВИДНОСТИ БАНКОВСКОГО СЕКТОРА}

\section{Аннотация:}

В статье рассматривается эффективное управление ликвидностью единого счета федерального бюджета в условиях невозможности размещения средств на банковских депозитах. Особенностью данной работы является актуализация применения рыночных мер минимизации рисков к управлению общественными фонансами. Доказывается, что существующие ограничения, связанные с безусловным исполнением принятых обязательств, могут быть сняты при помощи придания инструментам управления рыночного характера. Операции по управле нию средствами исследуются с позиций как казначейства, так и коммерческих банков, что позволяет демонстрировать значимые условия, по которым банки готовы реализовывать сделки, и естествен ные ограничения со стороны казначейства. Предлагается вариант управления, предусматривающий оптимальное решение между повышением доходности и снижением рискованности осуществляемых операций. Рассмотрена сегодняшняя ситуация размещения средств и сделан вывод о невозможности продолжения текущей политики в рамках избыточной ликвидности рынков и необходимости диверсификации операций по размещению. Представлены пути перехода к новым инструментам управления ликвидностью. Обоснована важность реформирования применяемых механизмов для повышения их привлекательности и доходности.

Ключевые слова:

ликвидность, управление ликвидностью, профицит ликвидности, казначейство, единый казначейский счет, доходность, риск, кассовое обслуживание.

\section{Zhantimirov Pavel Amambaevich}

Student, Department of Public Finances Financial University under the Government of the Russian Federation

\section{BALANCE MANAGEMENT ON THE SINGLE ACCOUNT OF THE FEDERAL BUDGET IN THE CONTEXT OF LIQUIDITY SURPLUS OF THE BANKING SECTOR}

\begin{abstract}
Summary:
The study discusses the effective liquidity management of the single account of the federal budget in the context of inability to deposit money in a bank. The novelty of this research is to update the market-based measures to minimize risks to the public finances management. The study proves that the current restrictions related to the strict fulfillment of the assumed obligations can be removed by making management tools market-oriented. Fund management is discussed from the standpoint of both Treasury and commercial banks. This approach demonstrates the essential terms for commercial banks to effect a transaction and natural restrictions imposed by the Treasury. The author proposes a management option providing an ideal solution for increasing the profitability and reducing the risks of such transactions. The present situation of the invest ment of funds is considered. It is concluded that the current policy should be changed in the context of excessive market liquidity. At the same time, the investment of funds is to be diversified. The transition to new tools of liquidity management is presented. The tools applied are to be reformed in order to increase their at tractiveness and profitability.
\end{abstract}

Keywords:

liquidity, liquidity management, liquidity surplus, Treasury, Single Treasury Account, profitability, risk, cashier services.

В настоящее время наблюдается активная трансформация отдельных областей управления общественными финансами, сопровождающаяся внедрением и использованием новых фринансовых инструментов, адаптацией механизмов корпоративного сектора к реалиям деятельности органов государственной власти. Безусловно, существенному реформированию подлежат процессы, связанные с кассовым обслуживанием исполнения бюджетов бюджетной системы Российской Федерации. В свою очередь значимая роль отводится Федеральному казначейству в сорере управления ликвидностью единого счета федерального бюджета [1, с. 4]. В условиях тесного переплетения механизмов корпоративного и государственного секторов происходит пересмотр целевых ориентиров кассового обслуживания. Наряду с обеспечением высокой мобильности и своевременности бюджетных платежей немаловажной целью качественного кассового обслуживания становится высокодоходное управление ликвидностью единого казначейского счета [2, с. 24]. На текущий момент список операций, осуществляемых со свободными остатками, постоянно расширяется, а качество управления данными потоками перманентно возрастает, о чем свидетельствуют показатели доходности реализуемых инструментов. Однако имеются зоны роста, которые позволят осуществлять управление на еще более высоком уровне. 
Сегодня одними из наиболее распространенных, устоявшихся и приемлемых с точки зрения соответствия предельным уровням риска являются операции по размещению временно свободных средств бюджета на банковских депозитах. Подобные операции необходимо рассматривать с позиций их участников. С одной стороны, такие инструменты служат источником фринансирования деятельности коммерческих банков. Для них это по сути дополнительный источник запаса ликвидности, альтернативный средствам рынка межбанковского кредитования, и сумм, привлекаемых от Центрального банка. Такая трактовка позволяет ориентироваться на требования по размещению, которые банки готовы принимать, в подобном случае анализируется спрос на размещаемые ресурсы. С другой стороны, это способ получения казначейством дополнительного дохода. Данный аспект позволяет оценивать действия Федерального казначейства по управлению ликвидностью, выбор из альтернативных вариантов размещения, а также возможные и реальные показатели доходности.

В периоды дефицита ликвидности банковского сектора средства федерального бюджета, размещаемые на счетах в банках, выступают значимым элементом финансовой устойчивости. В подобные моменты указанные суммы позволяют решать краткосрочные вопросы, связанные с нормативами ликвидности, возникающими кассовыми разрывами. Кроме того, если ставки по привлечению таких средств установлены ниже, чем на других рынках, данные суммы являются полноценными источниками для реального проведения финансовых операций, а не только в рамках покрытия образующегося дефицита. Для этого казначейству следует проводить работу как по управлению процентными ставками, так и по увеличению сроков операций, реализуемых с бюджетными средствами. Последняя мера способствует повышению доходности от сделок, поскольку они становятся привлекательнее для коммерческих банков с точки зрения устойчивости приобретаемой ликвидности.

Сегодня одной из важнейших характеристик размещения средств федерального бюджета является то, что такие операции достаточно стабильны, на эти средства действительно можно рассчитывать [3, с. 62]. Наибольший удельный вес занимают операции по размещению, которые не прерываются досрочно, а в условиях увеличения сроков банки могут значительно корректировать показатели ликвидности, повышая доходность по данным сделкам.

Однако могут возникать ситуации, когда в банковской отрасли наблюдается избыток ликвидности. Соответственно, подобные периоды сопровождаются действиями Центрального банка по стимулированию поиска альтернативных направлений инвестирования средств, что характеризуется снижением ставок по депозитным аукционам Банка России и ненадобностью казначейских денег в качестве источников фринансирования. Кредитные организации переориентируют внимание на активные операции. При этом от управления ликвидностью единого казначейского счета ожидается переход на другие направления размещения, что представляет большую проблему. Рынок предъявляет спрос на разные финансовые инструменты, однако крен в сторону высоколиквидных и низкодоходных банковских депозитов не позволяет мобильно управлять излишней ликвидностью, получать от данного шага дополнительные доходы. Рассмотренная ситуация представлена на рисунке 1.

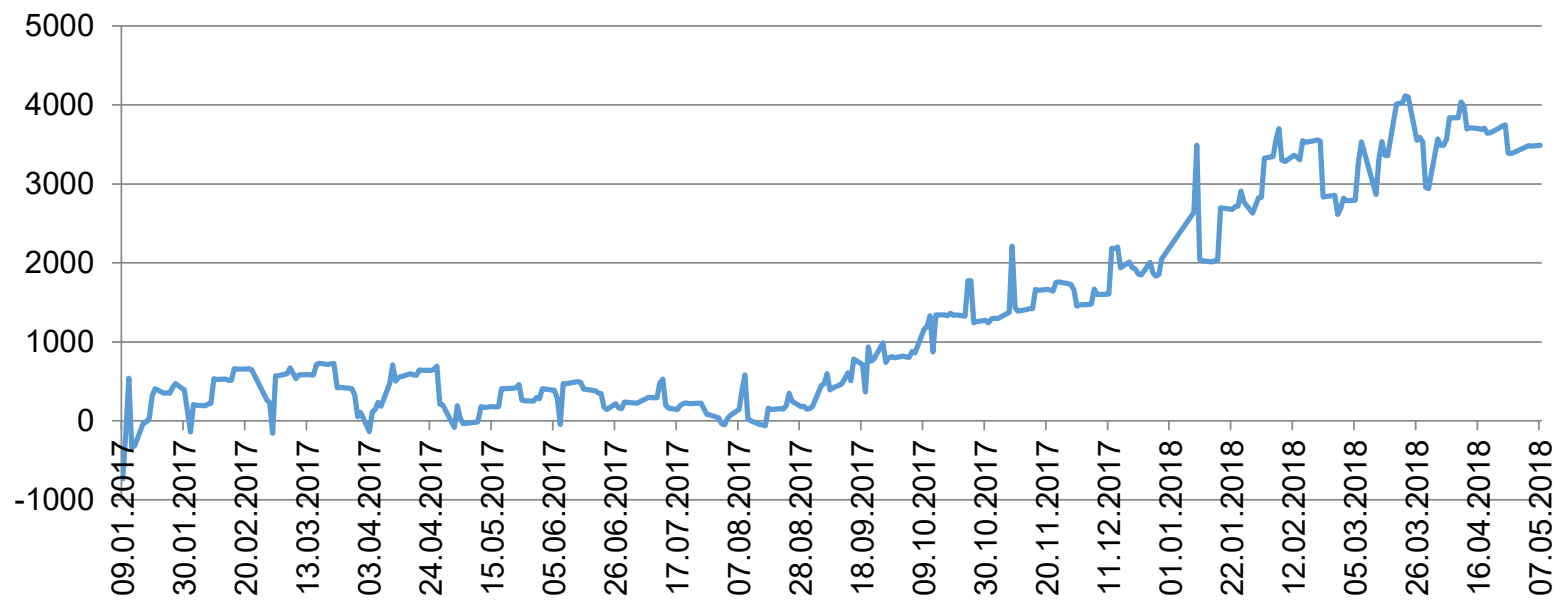

Рисунок 1 - Показатели ликвидности банковского сектора России в 2017-2018 гг. [4]

Сегодня мы можем наблюдать значительный профицит ликвидности в банковском секторе, который непосредственно отражается на размещении средств федерального бюджета. Ситуация 
является достаточно сложной с точки зрения поиска новых направлений вложения средств, поскольку депозиты имеют высокий удельный вес в общей сумме размещения в рамках управления ликвидностью. Поскольку актуальность подобных операций постепенно снижается, необходимо в данный период переориентироваться на сделки другого характера. Представленный график демонстрирует непосредственные объемы размещений и их связь с показателями ликвидности банковского сектора (рисунок 2).

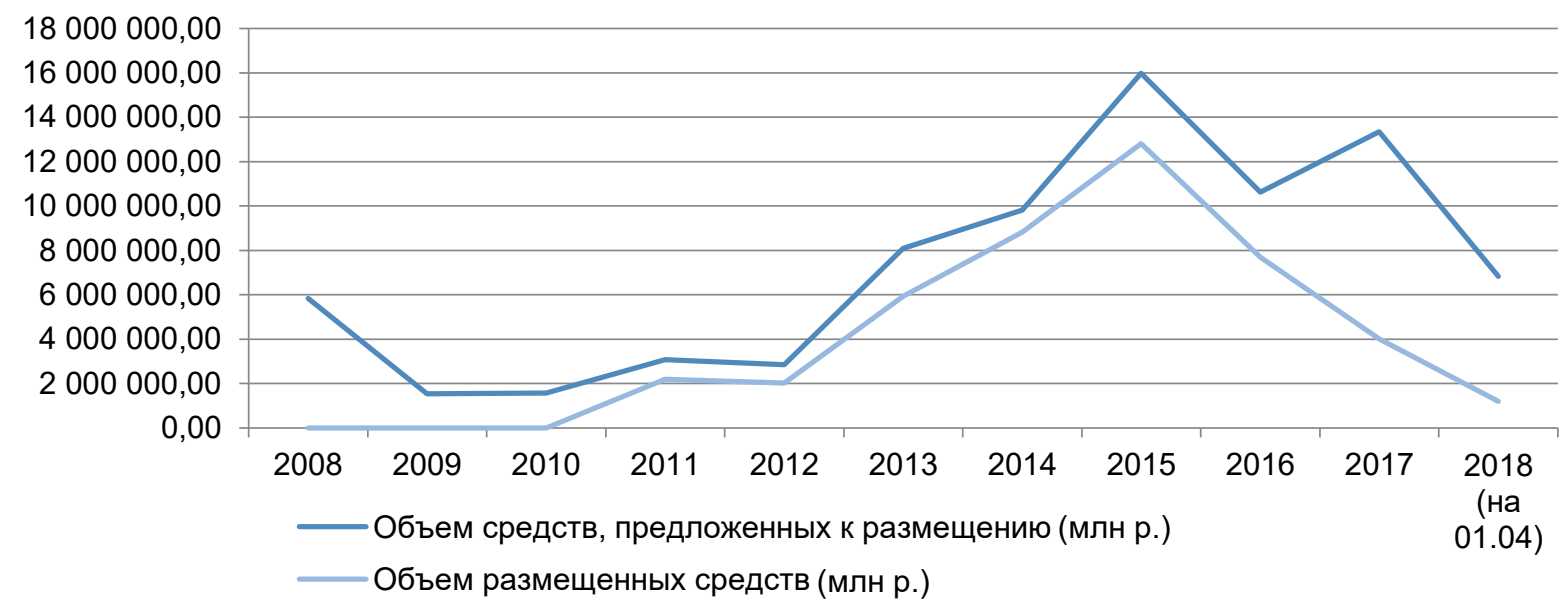

Рисунок 2 - Показатели размещения средств федерального бюджета на банковских депозитах в России в 2008-2018 гг. [5]

Указанные факторы воздействовали на изменения объемов средств, предлагаемых к размещению, что отражает верхняя линия на графике. Однако не менее важен уровень разрывов между предлагаемыми средствами и фрактически размещаемыми суммами. Отметим, что в отдельные периоды подобный разрыв практически не наблюдался, в то время как с 2014 г. он постоянно увеличивался, из чего можно заключить, что на размещение оказывали сильное влияние и внешние факторы, а не только характеризующие банковский сектор. Одним из них является состояние альтернативных источников фринансирования, которыми для коммерческих банков служат средства, привлекаемые от Центрального банка или рынка межбанковского кредитования. При прочих равных без учета преимущества в виде относительной стабильности и существенных объемов средств, размещаемых Казначейством, важной характеристикой следует назвать уровень процентных ставок, по которым данные сделки осуществляются. В таблице 1 отражены средние значения ставок по средствам, которые могут привлекать коммерческие банки.

Таблица 1 - Значения средних процентных ставок источников финансирования деятельности коммерческих банков в России в 2011-2017 гг. [6]

\begin{tabular}{|l|c|c|c|c|c|c|c|}
\hline \multicolumn{1}{|c|}{ Показатель } & $\mathbf{2 0 1 1}$ & $\mathbf{2 0 1 2}$ & $\mathbf{2 0 1 3}$ & $\mathbf{2 0 1 4}$ & $\mathbf{2 0 1 5}$ & $\mathbf{2 0 1 6}$ & $\mathbf{2 0 1 7}$ \\
\hline Ключевая ставка ЦБ & 8,13 & 8,17 & 8,25 & 9,50 & 12,80 & 10,25 & 8,75 \\
\hline Ставка MIACR & 3,79 & 5,66 & 6,19 & 8,00 & 13,30 & 10,44 & 9,09 \\
\hline Ставка по депозитам Казначейства & 5,88 & 6,70 & 5,85 & 9,94 & 12,21 & 10,36 & 8,44 \\
\hline
\end{tabular}

Мы придерживаемся мнения, что существенное влияние на управление процентными ставками в данное время оказали внешние шоки, а как следствие - таргетирование инфляции и валютного курса. Этот аспект принимается во внимание, однако в качестве главного рассматривается вопрос взаимосвязи политики Центрального банка и реакции коммерческих банков на привлекаемые бюджетные средства. Логика, которой придерживался ЦБ РФ при управлении ставками, реализуя сценарий абсорбирования ликвидности, заключалась в их постепенном повышении (одновременно наблюдались удорожание средств, привлекаемых от ЦБ, и повышение доходности сумм, размещаемых на его счетах). Однако ставки понижались в периоды относительной стабильности, что служило стимулом для размещения средств на счетах альтернативных источников финансирования. В соответствие с этим этапы повышения ключевой ставки были благоприятны для Казначейства в аспекте применения пониженных ставок и наращивания объемов реализуемых операций.

Однако стратегия в рассматриваемой области не может следовать только по пути увеличения доходности, поскольку должна быть увязана с целями денежно-кредитной политики. Данный курс был реализован в период с 2011 по 2014 г., когда отставание размещаемых сумм от 
предлагаемых к размещению было минимальным, так как предоставляемые источники были дешевле, чем источники Центрального банка и рынка МБК. С 2015 г. наблюдается ситуация снижения ставок и избыточной ликвидности, что определяет постоянное сокращение объемов размещаемых средств. Отметим, что казначейство при этом пытается принять контрциклические меры, наращивая объемы предложения и уменьшая уровень ставок значительно быстрее, чем изменяются альтернативные ставки. Однако они остаются незамеченными банковским сектором ввиду слишком сильного влияния профицита.

Очевидно, что моментальный переход к новой диверсифицированной модели невозможен, наиболее приемлемым представляется вариант с постепенным уравновешиванием всех видов операций по управлению ликвидностью. Одновременно с этим должны быть реализованы качественные изменения, касающиеся управления процентными ставками и сроками сделок. Увеличение длительности операций требуется лишь при условии качественных кассового планирования и прогнозирования [7]. Сегодня это одна из ключевых сложностей, поскольку при макроэкономической нестабильности нельзя точно определить величину и сроки выбытия средств. Решением данной проблемы в краткосрочном аспекте являются внедрение рыночных финансовых инструментов и увеличение количества операций на организованных торгах. Подобный шаг позволит изымать средства для погашения мгновенных обязательств без отрыва от направления размещения.

Все это подтверждает факт, что в нынешних условиях необходимы новые варианты размещения средств бюджета, которые отвечали бы запросам своевременного возврата, соблюдения предельных уровней рисков, но при этом обеспечивали бы сопоставимый с рыночным уровень доходности [8, с. 10]. В своем арсенале Казначейство РФ имеет ряд потенциально успешных инструментов, которые должны развиваться по аналогии с их применением в коммерческой или банковской среде. Альтернативным механизмом являются сделки репо, более привлекательные для коммерческих банков, поскольку могут использоваться для мгновенной конвертации ценных бумаг в денежные средства. Такой маневр позволяет в краткосрочной перспективе направлять финансы по другим инвестиционным сегментам, а после реализации второй части сделки - снова владеть указными инструментами.

Сегодня можно сказать, что казначейские договоры репо мало отличаются от операций по размещению средств на банковских депозитах, что определяет высокую занормированность инструмента. Однозначно, сделки репо со средствами федерального бюджета составляют существенную часть рынка данных механизмов, однако существует потенциал роста, позволяющий увеличивать совокупные объемы размещения и доходности. Доля реализованных сделок от числа рассматриваемых составляет около 80 \%, значит, не на все средства, предлагаемые к размещению, наблюдается достаточный спрос. Наконец, сам процесс отбора кредитных организаций таков, что список коммерческих банков, привлекаемых для заключения договоров, резко сокращается под действием нормативов, которые не всегда оправданы соотношением риска и доходности - конъюнктурой рынка.

Исходя из действия указанных фракторов, данная форма размещения временно свободных средств бюджета должна быть либерализована. Либерализация заключается в осуществлении сделок на рыночной основе с опорой на сопоставимые показатели банковского и коммерческого секторов. В таких условиях на первый план выходит вопрос минимизации кредитного и рыночного рисков, которые должны устраняться исключительно рыночными методами, а не при помощи властных полномочий. Примером служат операции на рынке производных фринансовых инструментов с базисными активами, являющимися предметом сделок по договорам репо. Технические возможности для этого сейчас имеются, поскольку Казначейство готовится к выходу на открытый рынок.

Безусловному реформированию сегодня подлежит состав активов, по которым заключаются договоры репо. Размещение реализуется только в отношении облигаций федерального займа, однако оно может быть распространено и на другие ценные бумаги, что позволит нарастить объемы операций и доходность по ним. В частности, Центральный банк России проводит аналогичные сделки с ценными бумагами из ломбардного списка, среди которых имеются как государственные бумаги, так и «голубые фишки», соответствующие качественным показателям. Примером могут послужить сделки, осуществляемые с центральным контрагентом, удельный вес которых в общей сумме договоров репо постепенно повышается, что объяснимо внедрением новых инструментов снижения рисков при расширении круга операций. Данные ситуации демонстрируют, что состав ценных бумаг, покупаемых Казначейством в рамках первой части сделки, может быть расширен без усиления рисков неплатежеспособности, что позволит размещать средства на счетах большего количества коммерческих банков.

Одним из важнейших вопросов сегодня является форма, в которой поставляются активы по договорам репо, что связано недополучением доходов, возникающим от перехода прав соб- 
ственности на актив. Эта проблема может быть решена путем внесения изменений в руководящие законодательные акты, касающиеся форм активов и способов их передачи. Широкий диапазон действий предоставляют клиринговые сертификаты участия, которые подразумевают наличие постоянных активов и дополнительную маневренность для продавцов в рамках первой части сделок. В свою очередь придание маневренности манипуляциям с «залоговой частью» сделки в данном случае означает повышение привлекательности и доходности инструмента.

Можно прийти к выводу, что ликвидностью единого счета федерального бюджета необходимо управлять с помощью большего количества рыночных инструментов воздействия. Сегодняшняя ситуация сближает деятельность казначейства с банковскими операциями, а в этих условиях невозможно развитие сценария нормативного регулирования. Исходя из этого, набор механизмов управления ликвидностью должен постоянно расширяться, чтобы система не была парализована из-за закрытия одного из направлений инвестирования средств. Кроме удовлетворения внутренних потребностей, диверсификация реализуемых действий обеспечит повышение количества, объемов и доходности операций по размещению средств. Большие надежды возлагаются на осуществление Казначейством операций с производными финансовыми инструментами, которые позволили бы минимизировать риски рыночными методами, получать бо́льшую прибыль, а также формировать определенный уровень доверия к срочному рынку за счет присутствия на нем постоянных устойчивых источников фринансирования.

\section{Ссылки и примечания:}

1. Прокофьев С. Единый казначейский счет федерального казначейства: состояние и перспективы // Государственные финансы. 2010. № 11. С. 2-7.

2. Шубина Л. Управление ликвидностью единого казначейского счета и оценка рисков операций по управлению остатками средств на нем // Финансы. 2013. № 8. С. 23-27.

3. Шубина Л. Развитие операций по управлению остатками средств на едином счете федерального бюджета, осуществляемых Федеральным казначейством // Экономика и управление. 2017. № 1. С. 60-65

4. Составлено автором на основе данных ЦБ РФ.

5. Составлено автором на основе данных Казначейства России.

6. Составлено автором на основе данных ЦБ РФ и Казначейства России.

7. Gadot G., Dalmia S. Liquidity Management: Best Practices for Banks. Improving Cash Forecasting to Enhance Liquidity Management [Электронный ресурс] // Information week. Bank systems \& Technology. 2014. 6 Oct. URL: https://www.banktech.com/compliance/liquidity-management-best-practices-for-banks/a/d-id/1297050d41d.html (дата обращения: 04.06.2018)

8. Прокофьев С. Комплексное управление денежными потоками в сфере российских государственных финансов // Финансы. 2014. № 9. С. 7-12.

\section{References:}

Gadot, G \& Dalmia, S 2014, 'Liquidity Management: Best Practices for Banks. Improving Cash Forecasting to Enhance Liquidity Management', Information week. Bank systems \& Technology, October 06, viewed 04 June 2018, $<$ https://www.banktech.com/compliance/liquidity-management-best-practices-for-banks/a/d-id/1297050d41d.html>.

Prokofyev, S 2010, 'Single account of the federal budget: state and prospects', Gosudarstvennyye finansy, No. 11, pp. 2-7, (in Russian).

Prokofyev, S 2014, 'Integrated management of cash flows in the field of Russian public finances', Finansy, No. 9, pp. 7-12, (in Russian).

Shubina, L 2013, 'Liquidity surplus of a single treasury account and risk assessment of balance management', Finansy, No. 8, pp. 23-27, (in Russian)

Shubina, L 2017, 'Development of operations to manage balance on a single account of the federal budget carried out by the Federal Treasury', Ekonomika i upravleniye, No. 1, pp. 60-65, (in Russian). 